\title{
Primary renal ganglioneuroblastoma in an adult
}

\author{
R.P. Jalleh, P.L. Newman, ${ }^{1}$ M.T. Jenkins and D.E. Sturdy \\ Departments of Surgery and ${ }^{1}$ Pathology, Royal Gwent Hospital, Newport, Gwent, UK.
}

Summary: A rare case of primary renal ganglioneuroblastoma in a 68 year old female is presented. The authors believe this to be the first case report of this tumour arising in the kidney of an adult patient.

\section{Introduction}

Ganglioneuroblastoma represents one of the three histological types of a group of neoplasms which originate in the sympathetic division of the autonomic nervous system; the other two being neuroblastoma and ganglioneuroma. The tumour itself is composed of undifferentiated neuroblasts and mature ganglion cells. Although neural crest tumours form one of the most common solid malignancies in childhood, ganglioneuroblastoma is exceedingly rare in adults. ${ }^{1}$ We report a case of this tumour with its primary site in the kidney.

\section{Case report}

A 68 year old female presented with a 3-week history of a painless, rapidly enlarging right loin mass. There was no haematuria. The patient had been hypertensive for 14 years, this being controlled with prazosin. General examination of the patient was essentially normal and abdominal palpation revealed a firm, non-tender mass measuring $15 \times 20 \mathrm{~cm}$ in the right flank suggestive of gross renal enlargement. Blood investigations were within normal limits. Intravenous urogram showed that the right kidney was largely replaced by a soft tissue mass, with distortion of the pelvicalyceal system (Figure 1). Computed tomography demonstrated that the renal mass had infiltrated the anterior and posterior abdominal walls and had extended directly up to the liver. In addition, there was para-aortic lymphadenopathy with caval compression. These findings were confirmed at operation, the tumour being unresectable, allowing only biopsy.

Correspondence: R.P. Jalleh, F.R.C.S., Department of Surgery, Hammersmith Hospital, Du Cane Road, London W12 0NN, UK.

Accepted: 2 May 1990

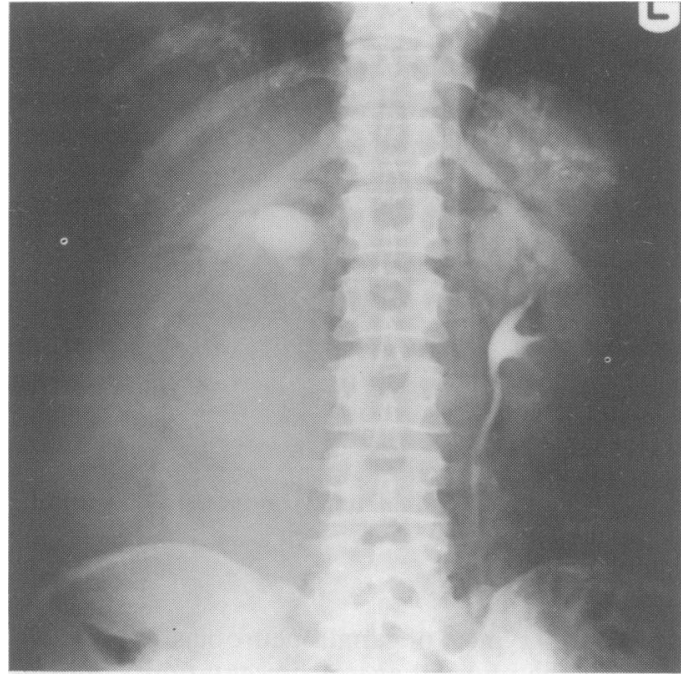

Figure 1 Intravenous urogram showing tumour mass compressing upper calyceal moiety of right kidney.

The biopsy material consisted of 5 tissue fragments. Histological examination of these revealed a tumour composed predominantly of small cells with round or oval hyperchromatic nuclei, showing no nucleoli and with very little cytoplasm. Scattered mitotic figures were present. In some areas, there was a fibrillary eosinophilic stroma. Occasional poorly developed rosette formation was seen. These represent a varying differentation of neuroblasts. In one of the fragments, there was an area of tumour containing larger cells with round open nuclei, prominent nucleoli and cytoplasm which was predominantly eosinophilic. These cells had the appearance of ganglion cells. There was some intermingling of these ganglion cells and neuroblasts (Figure 2). In the background tissue, occasional residual renal tubules were present (Figure 3). Histologically the tumour was 


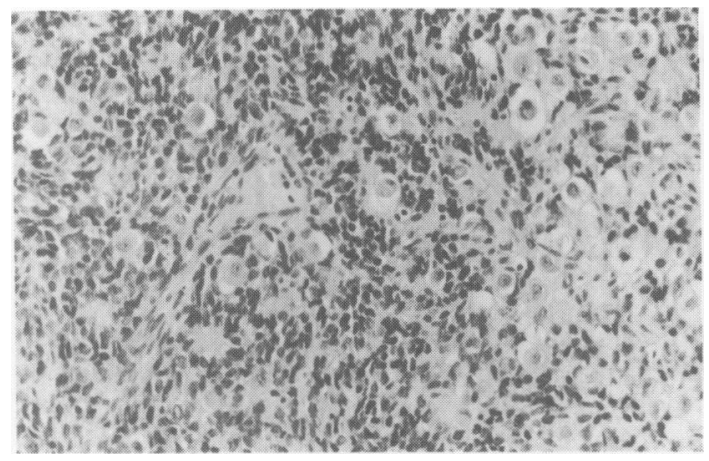

Figure 2 Tumour consisting of a mixture of small neuroblasts and larger nucleolated ganglion cells, with occasional rosettes $(\mathrm{H} \& \mathrm{E}, \times 105)$.

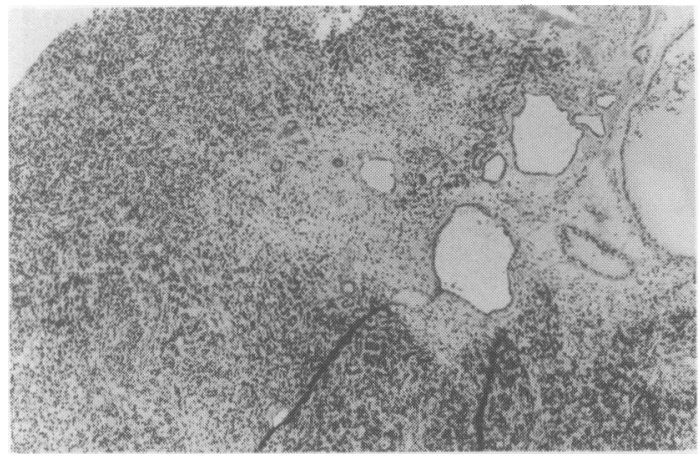

Figure 3 Some residual renal tubules present (H\&E, $\times 10.5)$.

considered typical of ganglioneuroblastoma, but in view of the unusual age of presentation, confirmatory evidence from immunocytochemistry and electron microscopy was sought.

Focal positive staining of both neuroblast and gangliocytic elements was obtained following reaction with antibodies to neurone specific enolase (NSE Dako), but no stromal staining was obtained following reaction with S-100 antibodies. The tissue was in less than optimal condition for electron microscopy, having been formalin fixed and paraffin embedded. However, sections were prepared and these showed the presence of neurosecretory granules, confirming the diagnosis of a neural tumour (Figure 4).

The dividing line between poorly differentiated ganglioneuroblastoma and neuroblastoma is in some instances rather subjective and may depend on adequate sampling. However, using the criteria of Adam and Hochholzer, ${ }^{2}$ in the presence of a mixture of mature ganglion cells and neuroblasts, we diagnosed ganglioneuroblastoma. The tissue samples were too small to accurately type the tumour, but in the classification of Stout, it prob-

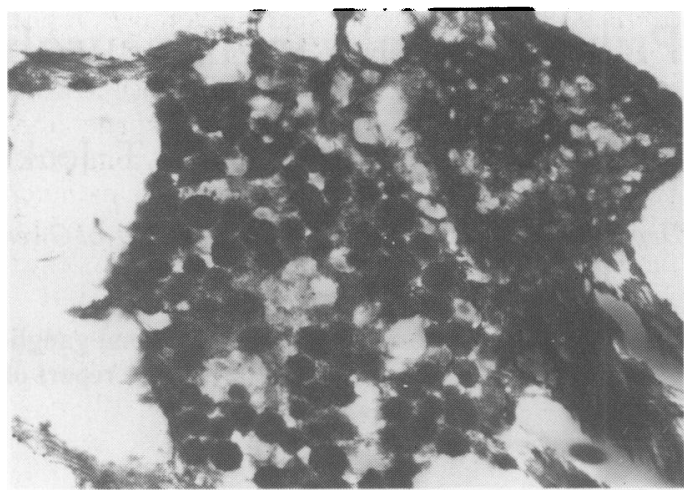

Figure 4 Electron micrograph of neurosecretory granules showing a central dense core with a peripheral halo $(\times 76000)$.

ably represents a composite or classical ganglioneuroblastoma. ${ }^{3}$

As the patient was largely asymptomatic and given the presence of a huge tumour bulk, no radiotherapy or chemotherapy was instituted. She died suddenly 3 weeks after laparotomy. Autopsy was not obtained.

\section{Discussion}

Ganglioneuroblastoma (GNB) is an extremely rare? tumour in adults. Only 25 cases have been documented in the literature in patients over the age of 20. In 1976, Kilton et al. described 2 cases and reviewed a further 17 from previously published reports. ${ }^{4}$ These did not include 5 cases from the records of the Armed Forces Institute of Pathology, as there was uncertainty as to whether these cases were included in the earlier descriptions. Since then, there have been a further 6 cases reported. ${ }^{2,5-7}$

The reported primary sites of this tumour include the mediastinum, neck and retro-peritoneum, although GNB has been identified to be arising from specific organs such as the adrenal gland ${ }^{4}$ and lung. ${ }^{6}$ The authors believe this to be the first reported case of primary GNB arising from the kidney in an adult patient. This is supported by the intravenous urogram where a definite calyceal moiety was demonstrated between the tumour mass and the site of the right adrenal gland. Further, histological examination of the biopsy specimen showed presence of residual renal tubules. It is postulated that adrenal rests, which have been described in the renal capsule, may represent a possible tissue of origin in this tumour.

The terminal outcome of this case was influenced by various identified unfavourable prognostic factors. Like that of neuroblastoma, prognosis in 
GNB is better in children 3 years or younger. It has also been noted that prognosis is much better in patients who have no evidence of metastasis at time of presentation. ${ }^{7}$ In our case, para-aortic and paracaval nodes were identified on computed tomography. There is also evidence that patients with tumours in extra-abdominal locations tend to fare better. ${ }^{2,4}$ Resectability of tumour is another favourable prognostic factor. Tumour free intervals of more than $2 \frac{1}{2}$ years have been reported in 2 cases where complete resection was possible., With regard to histology, the composite pattern as

\section{References}

1. Siegel, S.E. \& Sata, J.K. Neuroblastoma. In: Moosa, A.R. Robson, M.C. \& Schimpff, S.C. (eds) Comprehensive Textbook of Oncology. Williams and Wilkins, Baltimore, 1986, pp. $1211-1231$.

2. Adam, A. \& Hochholzer, L. Ganglioneuroblastoma of the posterior mediastinum: a clinicopathologic review of 80 cases. Cancer 1981, 47: 373-381.

3. Stout, A.P. Ganglioneuroma of the sympathetic nervous system. Surg Gynecol Obstet 1947, 84: 101-110.

4. Kilton, L.J., Aschenbrener, C. \& Burns, C.P. Ganglioneuroblastoma in adults. Cancer 1976, 37: 974-983. seen in this case has a considerably less favourable prognosis than tumours showing the diffuse pattern. ${ }^{7}$

\section{Acknowledgements}

The authors wish to thank Ms A. Bowlder of the Electron Microscopy Department of the University of Wales College of Medicine for the electron micrograph, and Mrs Marianne Jones of the Royal Gwent Hospital, Newport for her secretarial assistance.

5. Feigin, I. \& Cohen, M. Maturation and anaplasia in neuronal tumours of the peripheral nervous system with observation on glial-like tissue in the ganglioneuroblastoma. J Neuropathol Exp Neurol 1977, 36: 748-763.

6. Cooney, T.P. Primary pulmonary ganglioneuroblastoma in an adult: maturation, involution and the immune response. Histopathology 1981, 5: 451-463.

7. Talerman, A. \& Gratama, S. Primary ganglioneuroblastoma of the anterior mediastinum in a 61 year old woman. Histopathology 1983, 7: 967-975. 